\title{
New Lipid-Lowering Drugs: The Potential for Further Reductions in Cardiovascular Events
}

\author{
Henry W. Huang* \\ Division of Cardiovascular Medicine, Keck School of Medicine, University of Southern California, USA
}

The advent of effective pharmacologic therapies for cardiovascular disease has resulted in marked reductions in cardiovascular morbidity and mortality in industrialized nations. Statin drugs (HMG-CoA reductase inhibitors) have played a major part in this success. By reducing hepatocyte sterol synthesis, and thereby increasing hepatocyte uptake of LDL, statins cause a lowering of circulating LDL cholesterol levels and thus reduce the substrate for atherosclerotic plaque formation.

Although statins have been successful in reducing rates of cardiovascular events in patient populations, there remains a large burden of residual cardiovascular risk. Furthermore, statins are not uniformly well tolerated or effective in every individual patient. Some patients are not able to reach target lipid goals on statins or combination therapies. Other patients have difficulty tolerating statins, usually because of myalgias, their most common side effect. There continues to be a search for additional safe and effective lipid-lowering drugs to modify atherosclerotic disease and reduce cardiovascular events.

The cholesteryl ester transfer protein (CETP) inhibitors initially seemed to be a promising class of drugs since they raised HDL cholesterol, and higher levels of HDL have been associated with lower cardiovascular risk. Unfortunately, clinical trial data have not supported their use. Torcetrapib, the initial drug in this class, actually increased mortality [1], possibly through increased angiotensin-aldosterone activation and hypertension. Subsequent CETP inhibitors, dalcetrapib and anacetrapib, did not have these effects. Nevertheless, in the recently published dalOUTCOMES trial [2], dalcetrapib failed to lower cardiovascular event rates compared to placebo in patients on standard medical therapy for recent acute coronary syndrome. Based on the available evidence thus far, CETP inhibition does not appear to be a productive approach to improving cardiovascular outcomes.

One of the novel classes of lipid-lowering drugs in development are the PCSK-9 inhibitors. The PCSK9 enzyme is involved in the endocytosis and degradation of the LDL receptor from the liver surface. Inhibition of PCSK9 increases the amount of LDL receptor available to remove LDL from the bloodstream, thus lowering LDL cholesterol levels. A number of phase 2 randomized trials of AMG 145, a human monoclonal antibody to PCSK9, have been published recently [3-6]. A phase 2 randomized trial of AMG 145 showed approximately $43-50 \%$ reductions in LDL in hypercholesterolemic patients when used as monotherapy [3]. In heterozygous familial hypercholesterolemic patients, who were already on statins, the administration of AMG 145 resulted in up to 55\% further reductions in LDL levels [4]. A phase 2 trial of AMG145 in stable hypercholesterolemic patients on statins showed $42-50 \%$ reductions in LDL when it was administered every 4 weeks [5]. In patients with statin intolerance, AMG 145 lowered LDL by approximately $41-51 \%$, and lowered LDL by approximately $63 \%$ when administered at maximum dose with the addition of ezetimibe [6]. AMG 145 has been delivered as a subcutaneous injection every 4 weeks and appears to be well-tolerated, with no significant difference in adverse event profile compared to placebo. Another PCSK9 inhibitor is also undergoing clinical trial testing with promising results $[7,8]$.

Other novel lipid-lowering drugs under development (lomitapide, mipomersen) have primarily been studied for use in patients with familial hypercholesterolemia. Both lomitapide and mipomersen received recommendations for homozygous familial hypercholesterolemia by the drugs advisory committee of the FDA in October 2012, and both are under FDA review. Lomitapide inhibits microsomal triglyceride transfer protein, which drives the production and secretion of lipoprotein particles. In a phase 3 study, lomitapide decreased LDL cholesterol levels by $\sim 40 \%$ in homozygous familial hypercholesterolemia patients [9]. It also decreases triglyceride levels and will be studied for use in familial hyperchylomicronemia patients. Lomitapide is administered as a daily oral medication. Potential adverse events include GI side effects, increased liver transaminases, and increased liver fat. On the other hand, mipomersen is an apolipoprotein B-100 anti-sense oligonucleotide, which allows it to bind to apolipoprotein B-100 mRNA, induce its selective degradation, and thus decrease the secretion of apo- $\mathrm{B}$ containing lipoproteins from the liver. It is administered as weekly subcutaneous injections and lowers circulating LDL-cholesterol by 25\% in homozygous familial hypercholesterolemia patients [10]. Possible side effects include increased liver transaminases, increased liver fat, injection site reactions, flu-like sytems, fatigue, and pyrexia.

In conclusion, several promising new lipid-lowering drugs have been studied in recent years. LDL lowering, rather than HDL-raising, remains the proven approach to modifying atherosclerotic disease and improving cardiovascular outcomes. At this time, the PCSK9 inhibitors appear to be the most promising novel drug class with the broadest potential for application in various patient populations. More clinical trial outcomes data is needed for the new lipid-lowering drugs. None of the novel drugs are likely to replace statins, which are backed by an overwhelming evidence base and tout a proven track record in reducing cardiovascular events. Nonetheless, the new lipid-lowering drugs may become additional valuable pharmacotherapeutic options, either as adjunctive therapy in patients for whom statins are not effective enough or alternative therapy in patients who cannot tolerate statins. As a consequence, there is hope that more patients in the future will be able to reach lipid-lowering goals to minimize their cardiovascular risk, and that this will translate into further reductions in cardiovascular events around the world.

\section{References}

1. Barter PJ, Caulfield M, Eriksson M, Grundy SM, Kastelein JJ, et al. (2007) Effects

*Corresponding author: Henry W. Huang, Assistant Professor of Clinical Medicine, Division of Cardiovascular Medicine, Keck School of Medicine, University of Southern California, 1520 San Pablo St., Suite 1000, Los Angeles, CA 90033, USA, Tel: 323217-7491; E-mail: henryhua@usc.edu

Received November 24, 2012; Accepted November 25, 2012; Published November 27, 2012

Citation: Huang HW (2013) New Lipid-Lowering Drugs: The Potential for Further Reductions in Cardiovascular Events. Cardiol Pharmacol 2:e107. doi:10.4172/2329-6607.1000e107

Copyright: (c) 2013 Huang HW. This is an open-access article distributed under the terms of the Creative Commons Attribution License, which permits unrestricted use, distribution, and reproduction in any medium, provided the original author and source are credited. 
Citation: Huang HW (2013) New Lipid-Lowering Drugs: The Potential for Further Reductions in Cardiovascular Events. Cardiol Pharmacol 2:e107. doi:10.4172/2329-6607.1000e107

Page 2 of 2

of torcetrapib in patients at high risk for coronary events. N Engl J Med 357: 2109-2122.

2. Schwartz GG, Olsson AG, Abt M, Ballantyne CM, Barter PJ, et al. (2012) Effects of dalcetrapib in patients with a recent Acute Coronary Syndrome. N Engl J Med.

3. Koren MJ, Scott R, Kim JB, Knusel B, Liu T, et al. (2012) Efficacy, safety, and tolerability of a monoclonal antibody to proprotein convertase subtilisin/kexin type 9 as monotherapy in patients with hypercholesterolemia (MENDEL): a randomized, double-blind, placebo-controlled, phase 2 study. Lancet.

4. Raal F, Scott R, Somaratne R, Bridges I, Li G, et al. (2012) Low-density lipoprotein cholesterol-lowering effects of AMG 145, a monoclonal antibody to proprotein convertase subtilisin/kexin type 9 serine protease in patients with heterozygous familial hypercholesterolemia: the reduction of LDL-C with PCSK9 inhibition in heterozygous familial hypercholesterolemia disorder (RUTHERFORD) randomized trial. Circulation 126: 2408-2417.

5. Giugliano RP, Desai NR, Kohli P, Rogers WJ, Somaratne R, et al. (2012) Efficacy, safety, and tolerability of a monoclonal antibody to proprotein convertase subtilisin/ kexin type 9 in combination with a statin in patients with hypercholesterolemia (LAPLACE-TIMI 57): a randomized, placebo-controlled, dose-ranging, phase 2 study. Lancet.
6. Sullivan D, Olsson AG, Scott R, Kim JB, Xue A, et al. (2012) Effect of a monoclonal antibody to PCSK9 on low-density lipoprotein cholesterol levels in statin-intolerant patients: The GAUSS randomized trial. JAMA

7. Stein EA, Gipe D, Bergeron J, Gaudet D, Weiss R, et al. (2012) Effect of a monoclonal antibody to PCSK9, REGN727/SAR236553, to reduce low-density lipoprotein cholesterol in patients with heterozygous familial hypercholesterolemia on stable statin dose with or without ezetimibe therapy: a phase 2 randomized controlled trial. Lancet 380: 29-36.

8. Roth EM, McKenney JM, Hanotin C, Asset G, Stein EA, et al. (2012) Atorvastatin with or without an antibody to PCSK9 in primary hypercholesterolemia. N Engl J Med 367: 1891-1900.

9. Cuchel M, Meagher EA, du Toit TH, Blom DJ, Marias AD, et al. (2012) Efficacy and safety of a microsomal triglyceride transfer protein inhibitor in patients with homozygous familial hypercholesterolemia: a single-arm, open-label, phase 3 study. Lancet.

10. Raal FJ, Santos RD, Blom DJ, Marias AD, Charng MJ, et al. (2010) Mipomersen, an apolipoprotein B synthesis inhibitor, for lowering of LDL cholesterol concentrations in patients with homozygous familial hypercholesterolemia: a randomized, double-blind, placebo-controlled trial. Lancet 375: 998-1006. 